\title{
Molecular Wire Interconnects: Chemical Structural Control, Resonant Tunneling and Length Dependence
}

\author{
MATHIEU KEMP $^{\mathrm{a}}$, VLADIMIRO MUJICA $^{\mathrm{b}}$, ADRIAN ROITBERG $^{\mathrm{c}}$ and MARK A. RATNER ${ }^{\mathrm{a}, *}$ \\ ${ }^{a}$ Department of Chemistry and Materials Research Center, Northwestern University, \\ Evanston, Illinois 60208-3113; ${ }^{\mathrm{b}}$ Universidad Central de Venezuela, Caracas, Venezuela; \\ ${ }^{\mathrm{c}}$ National Institute of Standards and Technology, Gaithersburg, MD
}

\begin{abstract}
Molecular wires have several promising features, that would appear to make them ideal for advanced interconnects in nanoscale electronic devices. We discuss several aspects of the linear and nonlinear conductance of molecular wire interconnects. Topics include energy dependence of molecular conductance, resonant tunneling behavior, control of conductance by molecular structure and geometry, length dependence including the tunneling regime energetics. Design rules using molecular interconnects will differ substantially from those with more standard, lithographically structured silicon interconnects. In particular, the dissipation mechanisms will differ, both tunneling and ballistic regimes should be available, coulomb blockade and staircase behavior will be observed (but under differing conditions) and fabrication of gate electrodes is a challenge.
\end{abstract}

Keywords: Molecular wire, interconnect, resonant tunneling, molecular electronics

\section{INTRODUCTION}

Early work in molecular electronics was largely speculative, because the appropriate fabrication and characterization methods were not available on the nanometer distance scale characteristic of molecular structures [1-7]. Feynman's remarks concerning fabrication from the bottom up (by molecular or atomic assembly) rather than from the top down (by masking/lithograph techniques) is the intellectual antecedent for much of this work [8].
The advent of molecular surface functionalization and self-assembly methods [9], coupled with synthetic organic and inorganic chemical methods for producing molecular wire structures reproducibly and in good yield [10, 11], has improved candidate structures for molecular wires. Development of both scanning probes (especially STM) and nanofabricated measurement structures such as mechanically controllable break junctions [12], nanopore enclosures [13] and assembled nanodot arrays [14] has permitted structural investigation

*Corresponding author. Tel.:847/491-5652, fax: 847/491-7713, e-mail: ratner@chem.nwu.edu. 
of individual molecules or assemblies at electrode interfaces, and deduction of conductances for single molecules $[1,12,15,16]$.

Within the past 18 months, individual molecules have been shown to exhibit resonant tunneling behavior [17], coulomb blockade and staircase behavior at room temperature [12], gate control of electronic currents $[17,18]$, long distance (micron) current carrying capability $[17,18]$, and rectification properties [19]. Coupled with related work on inelastic electron tunneling spectroscopy [20], on Langmuir-Blodgett film conductivity [21], and on the much larger area of conductive organic materials [22], this recent work constitutes an impressive knowledge base suggesting that the use of molecular wires as interconnects is practical and promising.

Important advantages of molecular wires include:

1. Size (characteristic radii of molecular wires are subnanometer, while length can vary from subnanometer to micron).

2. Preparation: synthetic chemistry allows a myriad of candidate structures, with differing rigidity, optical properties, band gaps, electronic couplings, vibronic couplings, chemical reactivity, optical behavior, etc. [11, 22].

3. Low Cost: most polymer prices are quoted per pound or per ton.

4. Tailorability and functionalization possibility: molecular wire structures can be varied using synthetic chemistry; attachment to electrodes can vary in bond strength and electronic coupling $[11,22]$.

While much of the interest in molecular electronics has been in device structures [1-7] (molecular rectifiers [19, 23, 24], molecular logic gates and switches [25], molecular two-state devices or $n$ state devices, molecular recognition sensors [26], molecular optoelectronic gates), it is probably the interconnect structure of molecules that is most promising for hybrid molecular/solid state electronics.

\section{MOLECULAR WIRE CONDUCTANCE}

Electron transfer through molecular wires is closely related to intramolecular electron transfer, in which a molecular bridge is used to facilitate transfer of a single electron between terminal donor and acceptor molecular species [27, 28]. The difference between these two situations is that the continuum, that causes linear transport (rate constant in the intramolecular electron transfer situation or conductance in the molecular wire) will differ: for intramolecular electron transfer, the continuum is vibronic, while for molecular wires, the continuum is the electronic energy level continuum of the electrodes [27].

A characteristic molecular wire structure is shown in Figure 1A, in which a thiol terminated aromatic molecular bridge is linked to gold

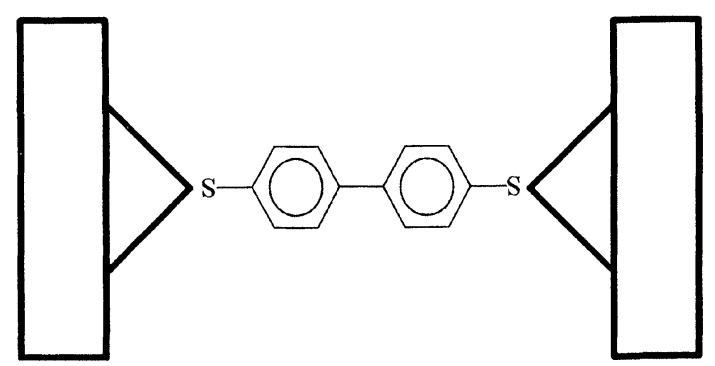

FIGURE 1A Schematic of a molecular wire group bridge between two continuum metal electrodes.

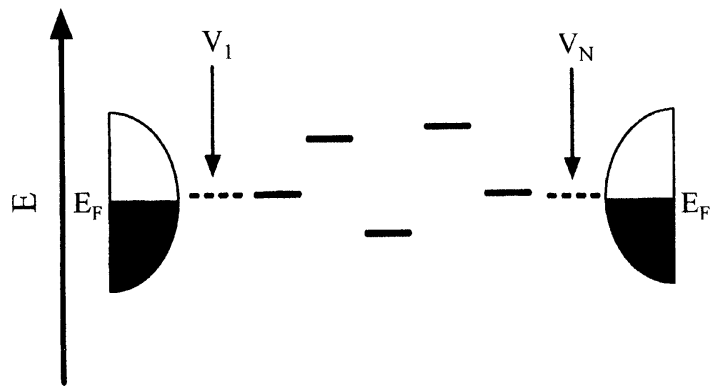

FIGURE 1B Energy level schematic diagram of a molecular wire structure like that in Figure 1a. The hemispherical structures represent the energy bands of the metal; $E_{F}$ is the Fermi energy. The discrete energy levels are those of the site orbitals in the molecule, while $V_{1}$ and $V_{N}$ are the matrix elements coupling the terminal sites in the molecular wire to the metallic interface. 
electrodes; in the sketch, the electrode link is indicated as a single interaction, but its actual nature will vary depending on the chemical binding or (in less favorable cases) physisorption interaction between bridge and electrode. The model that we will use for computing the conductance is sketched in Figure 1B. This is an energylevel diagram showing the continuum electrode levels, the fermi energy, the discrete energy levels corresponding to the atomic sites in the molecular wire, and the characteristic tunneling strengths (electronic mixing matrix elements) $V_{1}$ and $V_{N}$ at the first and $N$ th sites of the wire.

Several formalisms for calculating the conductance are available. Lang has developed [29] the density functional approach using a jellium model, and has demonstrated its ability to deal with atomic wire structures. Joachim and collaborators [30] have used an approach in which the electrode is represented as a tight binding, finite metal block. Datta and collaborators [31], and our group, have utilized a formalism in which the interaction between the discrete states of the molecular wire and the continuum states of the electrode is represented by a Newns/Anderson formalism [32]. Details are given elsewhere [33], but the important point is that, due to the bridge, the first and last sites in the molecule acquire a self-energy, denoted $\Sigma$. That is,

$$
\begin{aligned}
& H_{11} \rightarrow H_{11}+\sum_{1}(E) \\
& H_{N N} \rightarrow H_{N N}+\sum_{N}(E)
\end{aligned}
$$

Molecular site energies are unchanged, except for the first and last sites, each of which acquires a real part (energy shift) and an imaginary part (lifetime). Equation 2 indicates the two parts of the self-energy.

$$
\sum_{k}(E)=\wedge_{k}(E)-i \Delta_{k}(E) \quad K=1, N .
$$

Using the results of this discrete/continuum interaction representation, and the Bardeen tunneling formula [34], for conductance, one can derive formulas for the conductance $\mathrm{g}$ or current $\mathrm{I}$ both in the linear regime (current assumed proportional to voltage) and in the more general situation. These results are given by, respectively,

$$
\begin{gathered}
\mathrm{g}=\frac{2 e^{2}}{\pi \hbar} \Delta_{1}\left(E_{F}\right) \Delta_{N}\left(E_{F}\right)\left|G_{I N}\left(E_{F}\right)\right|^{2} \\
\mathrm{I}=\frac{2 e}{\pi \hbar} \int_{E_{F}-e W}^{E_{F}} d E \Delta_{1}(E) \Delta_{N}(E+e W)\left|G_{I N}(E, W)\right|^{2}
\end{gathered}
$$

Here $E_{f}$ is the electrode Fermi level (injection level), $W$ is the applied voltage, $4 \gamma$ is the bandwidth, $\Delta_{N}$ and $\Delta_{1}$ are the imaginary parts of the self-energy from Equation 2, given by

$$
\Delta_{k}(E)=V_{k}^{2} / \gamma\left\{\begin{array}{cc}
\sqrt{1-E^{2} / 4 \gamma^{2}} & |E / 2 \gamma|<1 \\
0 & |E / 2 \gamma|>1
\end{array}\right.
$$

Finally, $G_{1 N}$ is the $(1, N)$ matrix element of the electronic Green's function, described by

$$
G^{-1}(E)=E+i \eta+\sum(E)-H
$$

Here $H$ is the molecular electronic Hamiltonian, and $\eta$ is a positive infinitesimal.

The physical interpretation of Equation 3 is straightforward: the wire conductance is given by the atomic unit of conductance times the electrode interaction at the first and $N^{\text {th }}$ sites of the bridge, times the Green's function term that describes the electronic mixing between the orbitals at the two terminal sites of the molecular wire. Equation 4 generalizes this to the dependence upon applied voltage, and is applicable to the situation in which the source/drain current/voltage curve is not linear. This equation, thus, permits understanding of staircase behavior [33]. That requires, though, proper solution to the Poisson equation that describes the potential dependence of the molecular site energetics. This problem has not really been addressed yet in the published literature.

Although descriptions of the staircase behavior using Equation 4 have been given [33] we limit ourselves here to the linear conductance behavior, as the simplest characterization of the interconnect ability of these molecular wires. 


\section{STRUCTURAL AND ENERGETIC DEPENDENCE OF WIRE CONDUCTANCE}

Molecular wires will exhibit substantial sensitivity to the details of the molecular electronic structure. Understanding the dependence of conductance on laboratory parameters such as construction of the wire itself, temperature, chemical linkage between wire and electrode and injection energy requires a combination of chemical concepts for the wire and continuum concepts for the interface.

\subsection{Conductance Variation with Bridge Chemical Structure and Injection Energy}

Three continuous variables characterize the conductance of the molecular wire interconnect: the injection or Fermi energy, the temperature, and the potential difference between the two electrodes; for a linear conductance as given by Equation 3, the latter variable can be ignored. Temperature dependence is a complicated problem; one expects statistical factors in the electrodes and various shape modification of the barrier by the applied field (essentially the Fowler-Nordheim treatment) [35] and temperature dependent behaviors in the wire itself, including possible electron trapping behavior, dephasing, and structural changes. For simplicity, we neglect temperature effects (limit of low temperatures).

The only important continuous variable is then the injection energy. Figure 2 shows the predicted energy dependence for a fully conjugated (assumed planar) diphenyl bisthiol structure with gold electrodes. The notations $\sigma$ and $\pi$ refer to the angular momentum symmetries. The effective injection has both $\sigma$ and $\pi$ in planar geometries these are good symmetry quantum numbers, so that the channels can be separated. Several interesting aspects of Figure 2 can be noted: there is resonant tunneling behavior characterized by negative differential conductance - that is, as the

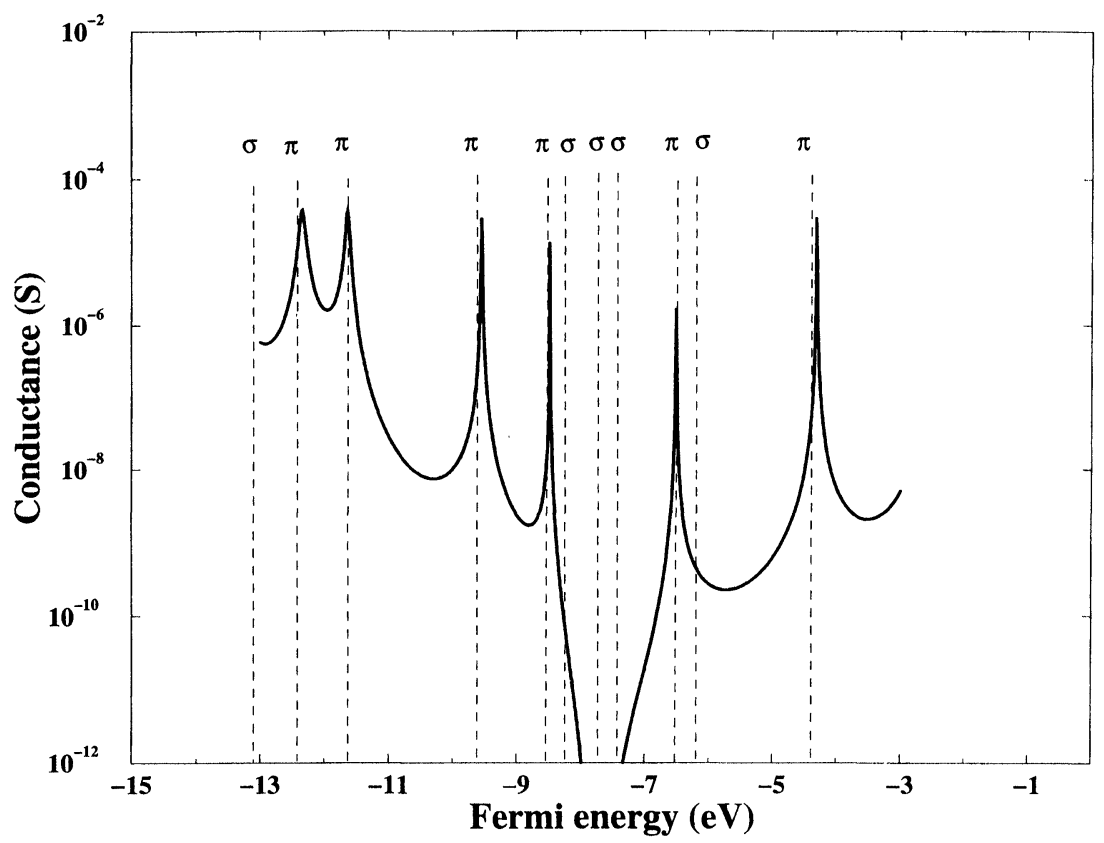

FIGURE 2 Conductance of the molecular structure in Figure 1A, calculated using the linear approximation formula of Equation 3. Note the resonances (--- lines represent molecular orbital eigenvalues). Resonant tunneling occurs whenever the $\pi$ level is degenerate with the Fermi energy. Conductance maxima are very close to the atomic unit of conductance, corresponding to efficient resonant tunneling. 
injection energy passes through one of the resonances corresponding to a molecular orbital of the bridging structure, the conductance exhibits a local maximum, subsequently decreasing with increased injection energy. The self-energy terms of Equation 2 become important here: divergence of the conductance is avoided because of the continuum structures of the electrodes, that provide imaginary (damping) behavior at the terminal sites of the molecular bridge. The finite conductance will be reduced compared to the atomic conductance $\mathrm{g}_{0}=(12.9 \mathrm{~K} \Omega)^{-1}$, by effects of molecular mixing, of the interfacial covalent link, and of vibrational dissipation that are not included in this discussion [40-42]. In general, the $\pi$-type contributions are larger than $\sigma$ : this is in accordance with a huge amount [36] of organic chemical wisdom, that delocalization through the $\pi$ system is much more effective than $\sigma$.

For actual conductance computation, it is important to know the injection energy. This problem is not yet solved, although it seems reasonable to assume [31,33] that the injection energy will be fixed at mid-gap. This assumption has been made in several computations, and agreement with experiment is generally very good. Actual proof requires self-consistent solution to the Poisson equation that describes actual electron density/potential structures in the inhomogeneous interconnect situation.

Since $\pi$-delocalization is generally stronger than $\sigma$, one might expect that twisting of the molecular structure to impede the $\pi$ conjugation would decrease conductance. This is shown in Figure 3 for triple bonded and double bonded systems respectively, and the differences are striking. The angular dependence is much smaller in the triple bond case, essentially because the triple bond is cylindrically symmetric. Conversely, the double bond exhibits mirror symmetry in the plane, so that twisting by $\pi / 2$ around it results in localization and strongly reduced conductance. The actual

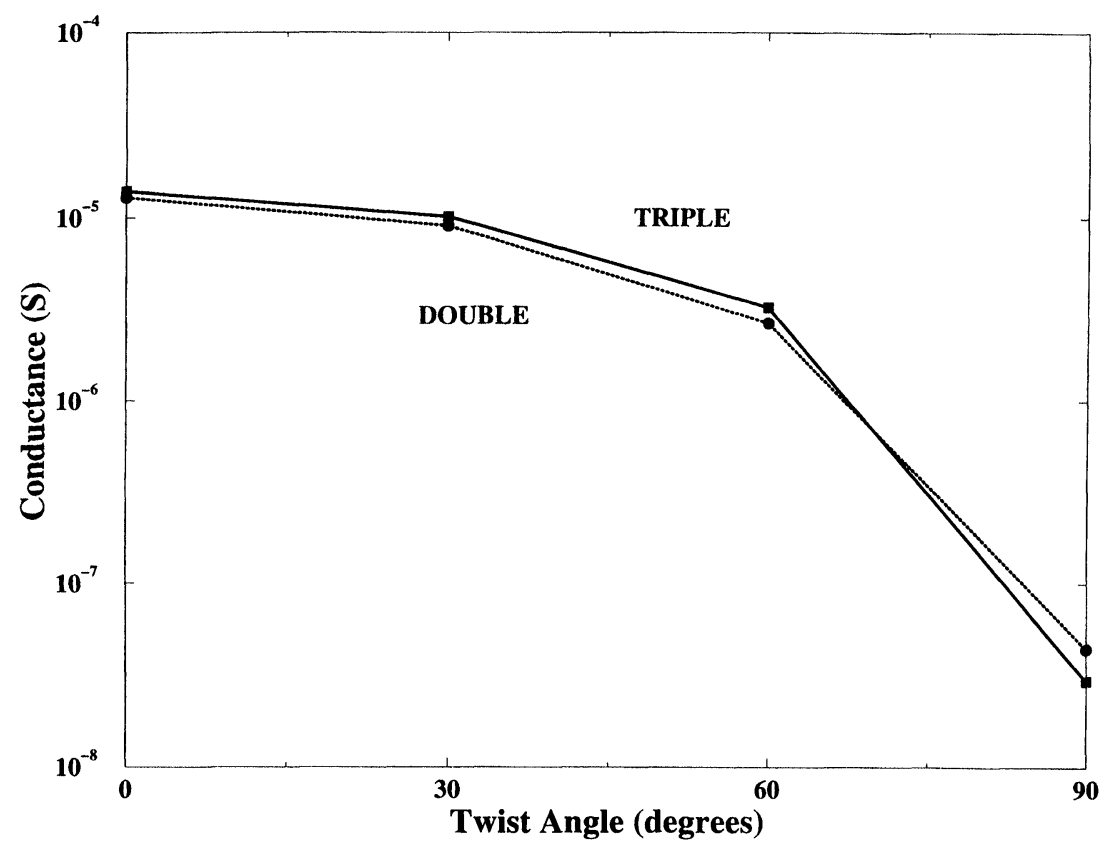

FIGURE 3 Computed conductance, as a function of twist angle, for molecules of the same structure as in Figure 1a, but with double and triple bonds inserted between the rings. When the $\pi$ systems become orthogonal ( 90 degree twist angle), the conductance is substantially reduced. The functional dependences on the double or triple bonds differ, because of the different symmetries involved. 
conductance of the double bonded structures are larger than that of the triple bonded bridges, because of bond alternation effects that result in stronger electron localization for the triple bonded (alkyne) than the double bonded (alkene) structures. Alkyne linkages are of interest in connection with molecular photonic wires [37] and nonlinear optical devices [38].

\subsection{Length Dependence - Tunneling and Ohmic Behaviors}

Molecular wires generally are not ohmic, except under special conditions. For an ohmic linear wire, one expects the conductance to scale as the inverse wire length. Exponentially-decaying tunneling has been well documented in intramolecular electron transfer reactions $[28,29]$, and is expected on a series of theoretical grounds to characterize molec- ular wires in the absence of dissipation, dephasing, trapping, and defect formation effects. Accordingly, Figure 4A shows the predicted conductance with thiol (sulfur) first and last chain sites and the indicated bridging units. Several characteristic behaviors expected on the basis of organic chemistry are apparent here: the $\pi$ systems are better conductors than the $\sigma$ systems, bond-length alternation effects make the alkynes less good than the alkenes, changing local bond geometries and so-called cis and trans linkages change the conductance of the polyalkyne structures.

Decay is exponential with length in all cases. The reason is that the electron is never actually localized on the molecular bridge, but rather undergoes bridge-assisted coherent tunneling between electrodes. This arises because in our formal approach, dissipation mechanisms, that would allow the electron actually to trap on the bridge, are not included.

\section{$\log 10$ (conductance) vs molecular length}

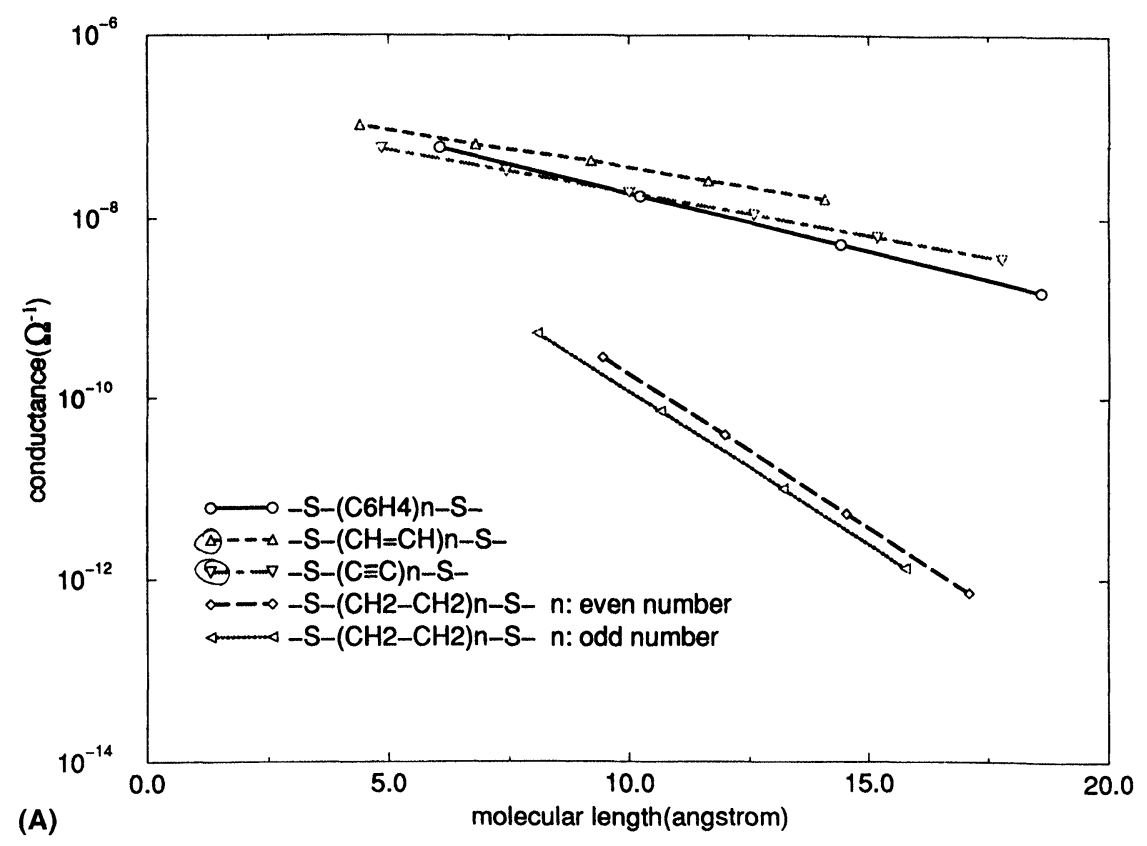

FIGURE 4A Logarithm of the conductance versus molecular length, for typical double bonded and triple bonded extended systems (such as those in Figure 1A, but with double bond or triple bond chains introduced between the rings). Notice the characteristic exponential decay with distance, and the different decays of the triple and double bonded systems. 
Inclusion of dissipation has been discussed for intramolecular electron transfer [40-42] (the rate constant for intramolecular electron transfer is expected to scale generally as the conductance for molecular wires, although inclusion of dissipation in real molecular wire calculations has not yet been presented). Figure 4B presents the length dependence of the intramolecular transfer rate if a tight binding model is assumed, and the matrix element $V_{b}$ characterizes the mixing between the sites of the molecular bridge of Figure 1. The gap, $\omega$, is the energy required to place an electron in an affinity level (that is, to reduce chemically) of the molecular bridge. For fixed mixing matrix element $V_{1}=V_{N}=V_{b}$, there is substantial energy variation of the rate constant. Indeed, when the gap becomes comparable to the mixing matrix element, there is essentially no decay of the rate constant with the length of the bridge (since the continuum is included, resonance tunneling does not result in a divergence of the rate constant). Physically, dissipation mechanisms will always occur, so that no true length independence can occur. In weak dissipation, however, the length decay may be very small, as has recently been observed $[17,18]$ in very low temperature measurements on carbon nanotube structures.

The energy gap $\omega$ of Figure 4B is related to the injection energy of Figure 2; vanishing $\omega$ corresponds to injection exactly at one of the resonances in Figure 2.

Functional dependence of the rate constant (conductance) can be formulated simply if $V_{b} / \omega$ is much less than one, and the tight binding model

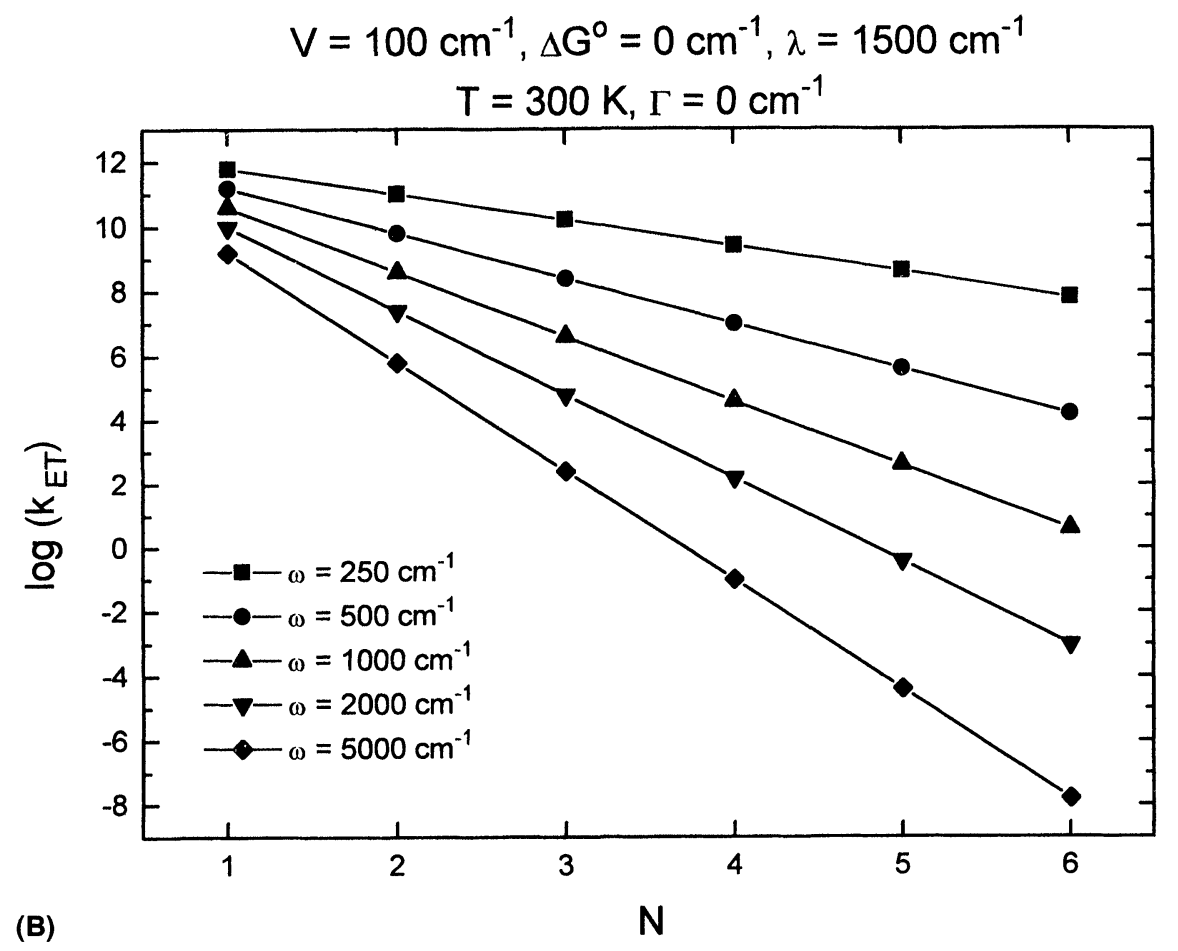

FIGURE 4B The calculated intramolecular electron transfer rate (units of $\mathrm{s}^{-1}$ ). The parameters are those of a simple tight binding model, as in Equation 7; $V$ is the matrix element between neighboring sites, $\lambda$ the reorganization energy, and $\omega$ the gap between donor or acceptor levels and bridge levels. Note that the characteristic decay parameter (the $\beta$ of Equation 9) indeed strongly depends on the gap size. 
(to chemists, the Huckel model) is assumed for the molecular bridge. Under these conditions, the Green's function will scale like [42] $(\hbar \equiv 1)$

$$
G_{I N} \sim \frac{V_{1} V_{N}}{\omega}\left(V_{B} / \omega\right)^{N-1}
$$

for an $N$-site bridge, and then the conductance behavior (or rate constant) is expected to follow Equation 8.

$$
g(N)=g(1) \exp \left\{-\beta(N-1) R_{0}\right\}
$$

This exponential decay is then characterized by the constant $\beta$, which in the simplest (super exchange) model is given as

$$
\beta=\frac{-2}{R_{0}} \ln \left(V_{B} / \omega\right)
$$

Here $R_{0}$ is the repeat unit length on the molecular bridge. Exponential behavior has been very well studied for intramolecular electron transfer rates $[28,43]$.

\section{REMARKS}

Molecules are of substantial interest as possible device components in advanced electronics and optoelectronics; their most obvious and promising short-term application is in the area of interconnects. Electron transfer can, then, be used directly to pass current among active device components (or, more speculatively, to provide field switching possibilities) [46].

We have analyzed several of the characteristic behaviors to be expected for single molecule interconnects. Even in relatively unfavorable cases, single molecules can carry a current of order $0.1 \mathrm{nA}$ for applied fields of one volt; $[14,15]$, this is large enough for applications, and can be increased in more favorable cases such as single-walled nanotubes [18] or strongly conjugated oligomers.

There are, essentially, two regimes in the conductance of characteristic molecular wires. At low temperatures, resonant tunneling structures are expected, with substantial increases in the conductance (approaching the atomic unit of conductance), near molecular resonances, suggesting possible resonant tunneling applications. With increasing temperature, dephasing processes will become important and one will eventually find ohmic behavior, with the conductance scaling inversely with length [42]. Because molecules are substantially smaller than the usual quantum dot structures, coulomb blockade and staircase effects can be seen [12] not only at low temperatures but also at room temperature, suggesting the possibility of single electronics under ambient conditions.

The work on molecular wires to date is at the proof of concepts stage: problems such as the relative resistance of particular molecular wire structures $[30,31]$, the specific attachment of molecular wires to electrode surfaces, understanding the temperature and length dependence of the conductance [33], and engineering the geometry of particular molecular wire interconnects [11] have been discussed. It is clear that use of molecular wires as circuit elements still requires overcoming several major obstacles, of which the most serious is probably addressing individual molecular wires, and anchoring them in a specific geometry on assembled chip structures.

\section{Acknowledgements}

We are grateful to the NSF and CONICIT for support through a collaborative NSF/CONICIT international research program. MR is also grateful to the chemistry divisions of the National Science Foundation and the Office of Naval Research for support. We are indebted to Mark Reed, Chongwu Zhou, Abe Nitzan, Bill Davis, Yi Mao and Mike Wasielewski for very helpful suggestions and enjoyable collaboration.

\section{References}

[1] F. L. Carter, ed. (1982). Molecular Electronic Devices, New York: Dekker.

[2] F. L. Carter, R. K. Siatkowski and H. Wohltgen, eds. (1988). Molecular Electronic Devices, Amsterdam: Elsevier. 
[3] R. R. Birge, ed. Molecular and Biomolecular Electronics, (ACS, Washington, DC, 1994)

[4] R. M. Metzger, P. Day and C. G. Papavassiliou, eds., (1991). Lower-Dimensional Systems and Molecular Electronics, New York: Plenum.

[5] A. Aviram, ed. (1992). Molecular Electronics-Science and Technology, Washington, DC: AIP.

[6] J. Jortner and M. A. Ratner, Eds., Molecular Electronics (Blackwells, London, 1997).

[7] M. C. Petty, M. R. Bryce and D. Bloor, ed. Introduction to Molecular Electronics, (Oxford, New York, 1995).

[8] Feynman, R. P. (1960). Eng. and Science, 23, 22 ; in H. D. Gilbert, Ed., Miniaturization (Reinhold, New York, 1961).

[9] Mirkin, C. A. and Ratner, M. A. (1992). Ann. Rev. Phys. Chem., 43, 719.

[10] Wu, C. G. and Bein, T. R. (1994). Science, 264, 157.

[11] Tour, J. M. (1996). Chem. Revs., 96, 537.

[12] Zhou, C., Muller, C. J., Reed, M. A., Burgin, T. P. and Tour, J. M., in ref. 6; Zhou, C., Reed, M. A., Muller, J., Burgin J. P. and Tour, J. M. submitted to Science.

[13] Zhou, C. and Reed, M. A., unpublished.

[14] Dorogi, M., Gomez, J., Osifchin, R., Andres, R. P. and Reifenberger, R. (1995). Phys. Rev. B., 52, 9071; Andres, R. P., Bein, T. and Dorogi M. et al. (1996) Science, 272, 1323.

[15] Joachim, C., Gimzewski, J. K., Schlitter, R. D. and Chavy, C. (1995). Phys. Rev. Lett., 74, 2102; Joachim, C. and Gimzewski, J. K. (1995). Europhys. Lett., 30, 409.

[16] Tao, N. J. (1996). Phys. Rev. Lett., 76, 4066.

[17] Bockrath, D. M., Cobden, D. H., McEuen, R. L., Chopra, N. G., Zettl, A., Thess, A. and Smalley, R. A. (1997). Science, 275, 1922 .

[18] Tans, S. J., Devoret, M. H., Dai, H., Thess, A., Smalley, R. J., Geerligs, L. J. and Dekker, C. (1997). Nature, 386, 474.

[19] Dhirani, A., Lin, P. H., Guyot-Sionnest, P., Zehner, R. W and Sita, L. A. (1997). J. Chem. Phys., 106, 5249.

[20] Hipps, K. W. and Mazur, U. (1994). J. Phys. Chem., 98, 5824; Mazur, U. and Hipps, K. W. (1994). J. Phys. Chem., 98, 8169.

[21] Fischer, C. M., Burghard, M., Roth, S. and Klitzing, K. V. (1994). Europhys. Lett., 28, 129; Fischer, C. M., Burghard, M. and Roth, S., in Ref. 6.

[22] W. R. Salaneck, I. Lundstrom and B. Ranby, ed. (1991). Conjugated Polymers and Related Compounds, London: Oxford University Press, 1993; J. L. Bredas and R. Silbey, eds. Conjugated Polymers., Dordrecht: Kluwer, 1991.

[23] Aviram, A. and Ratner, M. A. (1974). Chem. Phys. Lett., 29, 277.

[24] Metzger, R. M., in Molecular and Biomolecular Electronics, edited by R. R. Birge (ACS, Washington, DC, 1994), p. 81 a very complete overview.

[25] Walz, J., Ulrich, K., Port, H., Wolf, H. C., Wonner, J. and Effenberger, F. (1993). Chem. Phys. Lett., 213, 322; K. Schaumburg in W. Gopel and C. Ziegler, eds. (1992). Nanostructures Based on Molecular Materials, Weinheim: VCH, 156

[26] Mirkin, C. A., Ulrich, K., Mucic, R. C. and Storhoff, J. J. (1996). Nature, 382, 607; Alivisatos, A. P., Johnson, K. P., Peng, X., Wilson, P. E., Loweth, C. J., Bruchez, M. P. and Schultz, P. G. (1996). Nature, 382, 609.

[27] Ratner, M. A. and Jortner, J., in Ref.6.

[28] Marcus, R. A. and Sutin, N. (1985). Biochim. Biophys. Acta., 811, 265; Newton, M. D. and Sutin, N. (1984). Annu. Rev. Phys. Chem., 35, 437; Barbara, P. F., Meyer, T. J. and Ratner, M. A. (1996). J. Phys. Chem., 100, 13148.
[29] Lang, N. D. (1996). Phys. Rev. B., 52, 5335.

[30] Joachim, C. and Vinuesa, J. F. (1996). Europhys. Lett., 33, 635.

[31] Datta, S. (1995). Electronic Transport in Mesoscopic Systems, Cambridge: Cambridge University Press; Samanta, M. P., Tian, W., Datta, S., Henderson, J. I. and Kubiak, C. P. (1996). Phys. Rev. B., 53, 7626.

[32] Newns, D. M. (1969). Phys. Rev., 178, 1123; Anderson, P. W. (1961). Phys. Rev., 124, 41.

[33] Mujica, V., Kemp, M. and Ratner, M. A. (1994). J. Chem. Phys., 101, 6849; Mujica, V., Kemp, M. and Ratner, M. A. (1994). J. Chem. Phys., 101, 6856; Kemp, M., Mujica, V. and Ratner, M. A. (1993). J. Chem. Phys., 101, 5172; Mujica, V., Kemp, M., Roitberg, A. and Ratner, M. A. Condensed Matther Theories II, edited by E. V. Luduena, P. Vashishta and R. F. Bishop (Nova Science, Commack, 1996) (in press); Mujica, V., Kemp, M., Roitberg, A. and Ratner, M. (1996). J. Chem. Phys., 104, 7296.

[34] Landauer, R. (1957). IBM J. Res. Dev., 1, 223; Landauer, R. (1981). Phys. Lett., 85a, 91.

[35] Zhou, C. and Reed, M. A., Private Communication.

[36] Pope, M. and Swenberg, C. E., Electronic Processes in Organic Crystals (Oxford, 1982).

[37] Lindsey, J. M. (1996). J. Am. Chem. Soc., 11, 3996.

[38] Priyadarshy, S., Therien, M. J. and Beratan, D. N. (1996). J. Am. Chem. Soc., 8, 1504.

[39] Cave, R. and Newton, M. D., in ref. 6.

[40] Skourtis, S. and Mukamel, S. (1995). Chem. Phys., 197, 367.

[41] Felts, A. K., Pollard, W. T. and Friesner, R. A. (1995). J. Phys. Chem., 99, 2929.

[42] Davis, W., Wasielewski, M., Mujica, V., Nitzan, A. and Ratner, M. A. J. Phys. Chem., in press.

[43] Risser, S., Beratan, D. N. and Onuchic, J. N., in Ref. 6.

\section{Authors' Biographies}

Mathieu Kemp was born in Montreal, Canada in 1963. He received his Ph.D. in Physics from the University of North Carolina in 1992. His research interests include: molecular electronics, nanoelectronics, and electronic transport in amorphous semiconductors. He is working with Mark Ratner at Northwestern University.

Adrian Roitberg was born in Buenos Aires, Argentina in 1962. He received his Ph.D. in Chemistry form the University of Illinois at Chicago in 1992. His research interests include: protein modeling, electron transfer, and molecular electronics. $\mathrm{He}$ is a guest research chemist at the National Institute for Standards and Technology.

Vladimiro Mujica was born in Caracas, Venezuela in 1954. He received his Ph.D. in Quantum Chemistry form Uppsala University in 1985. His 
research interests include: mesoscopic devices, nonlinear optics and density functional theory. $\mathrm{He}$ is a professor at Universidad Central de Venezuela.

Mark A. Ratner was born in Cleveland, Ohio in 1942. He received his Ph.D. in Chemistry from
Northwestern University in 1969. His research interests include: nonlinear optics, electron transfer, molecular electronics, protein modelling, polymer electrolyte transport, and SCF methods. $\mathrm{He}$ is a professor at Northwestern University. 

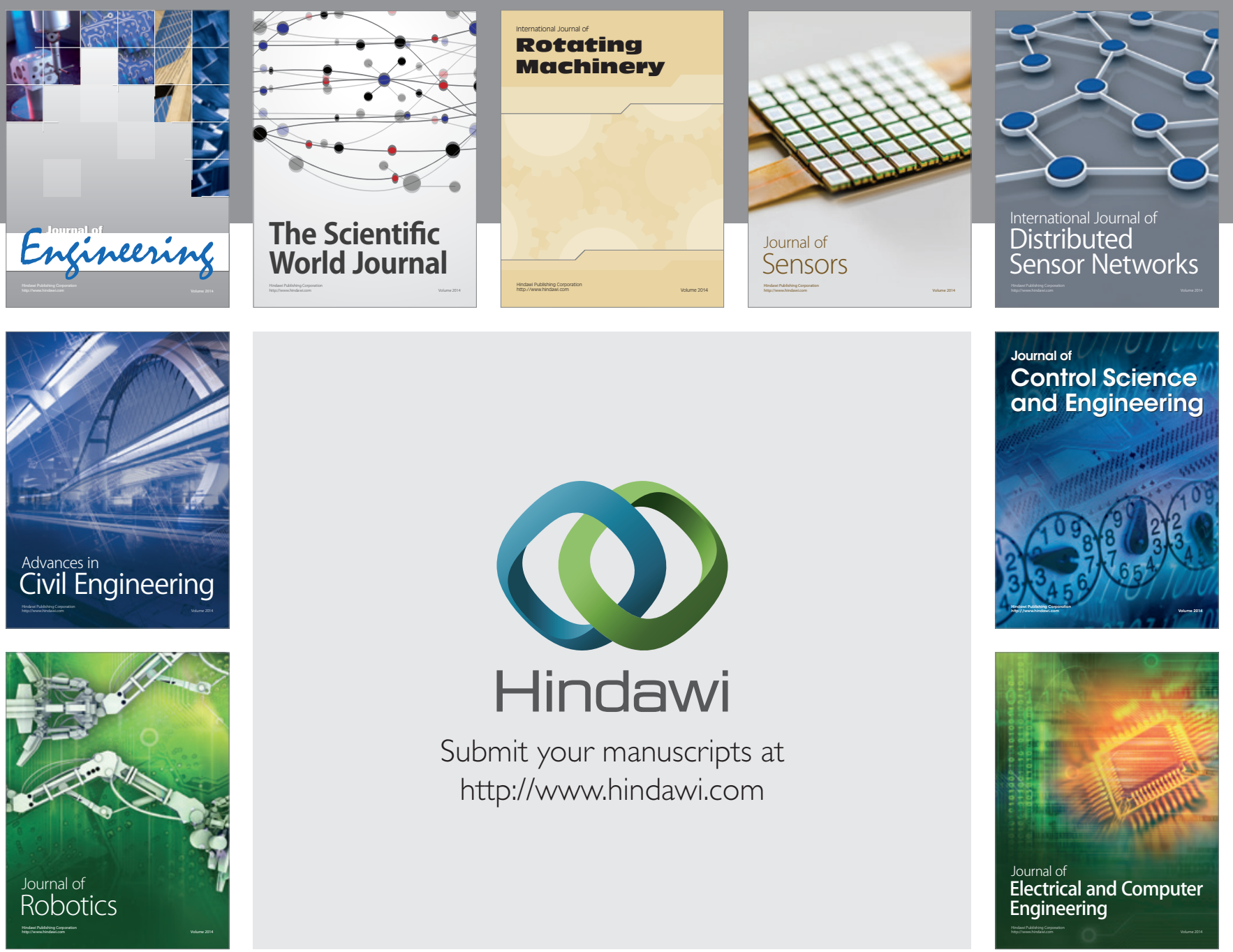

Submit your manuscripts at

http://www.hindawi.com
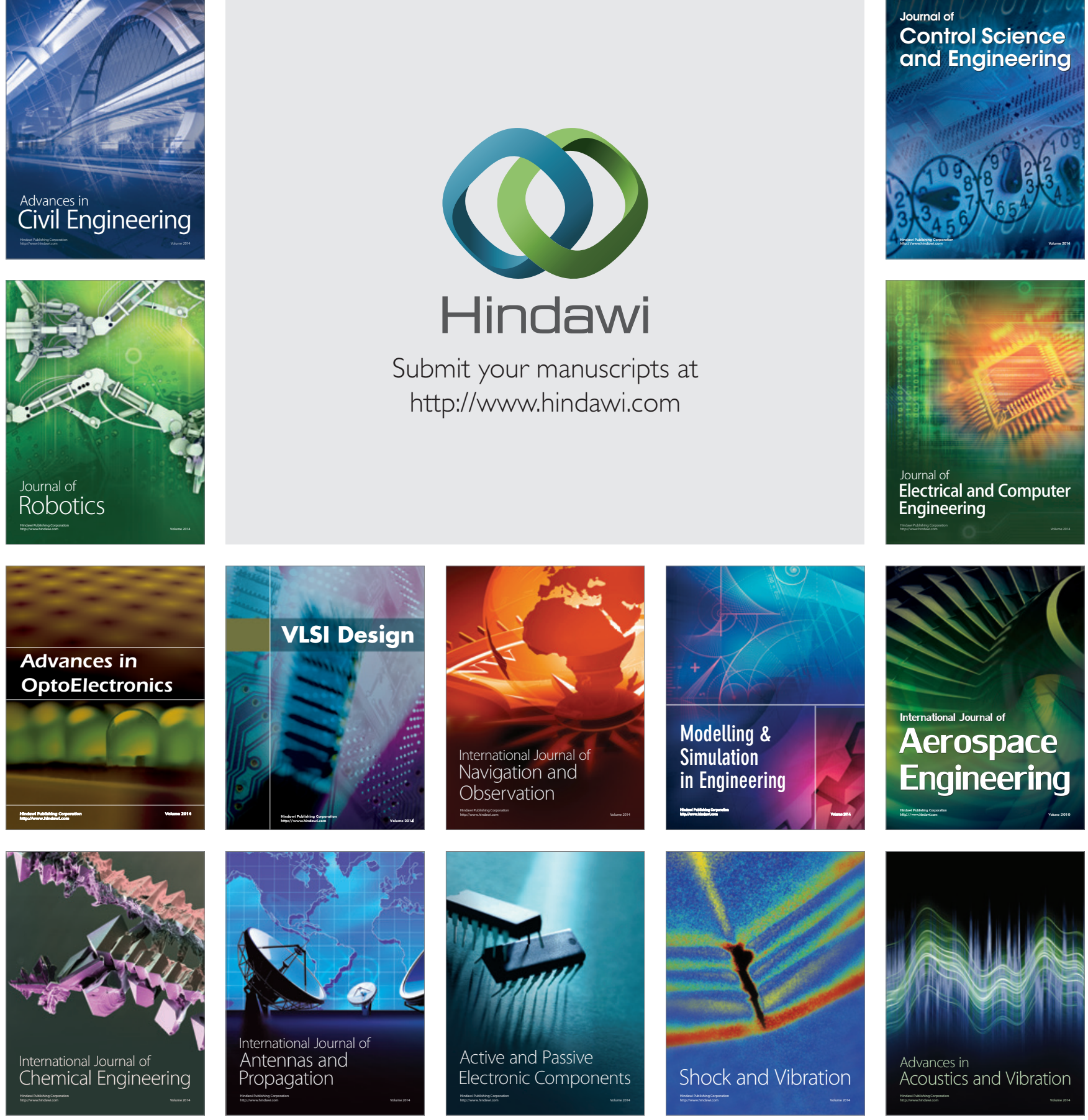